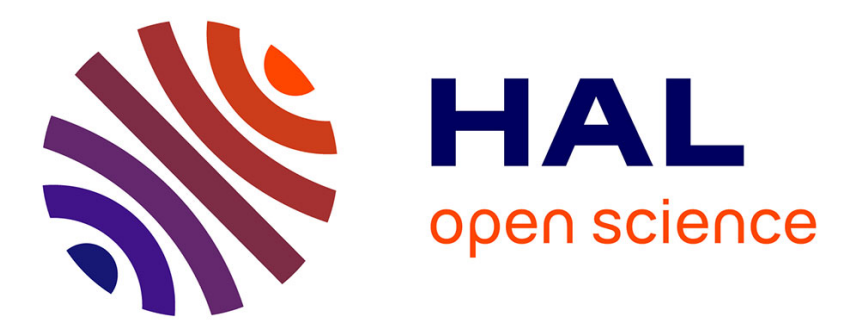

\title{
Adaptive Tone Reservation for better BER Performance in a Frequency Selective Fading Channel
}

Marwa Chafii, M Lamarana Diallo, Jacques Palicot, Faouzi Bader, Rémi

Gribonval

\section{- To cite this version:}

Marwa Chafii, M Lamarana Diallo, Jacques Palicot, Faouzi Bader, Rémi Gribonval. Adaptive Tone Reservation for better BER Performance in a Frequency Selective Fading Channel. IEEE VTC2016Spring, May 2016, Nanjing, China. 10.1109/vtcspring.2016.7504119 . hal-01290918

\section{HAL Id: hal-01290918 \\ https://hal-centralesupelec.archives-ouvertes.fr/hal-01290918}

Submitted on 19 Mar 2016

HAL is a multi-disciplinary open access archive for the deposit and dissemination of scientific research documents, whether they are published or not. The documents may come from teaching and research institutions in France or abroad, or from public or private research centers.
L'archive ouverte pluridisciplinaire HAL, est destinée au dépôt et à la diffusion de documents scientifiques de niveau recherche, publiés ou non, émanant des établissements d'enseignement et de recherche français ou étrangers, des laboratoires publics ou privés. 


\title{
Adaptive Tone Reservation for better BER Performance in a Frequency Selective Fading Channel
}

\author{
Marwa Chafii *, M. Lamarana Diallo *, Jacques Palicot *, Faouzi Bader *, Rémi Gribonval ${ }^{\dagger}$ \\ * CentraleSupélec, IETR, Campus de Rennes \\ 35576 Cesson - Sévigné Cedex, France \\ Email: marwa.chafii@supelec.fr \\ $\dagger$ Inria - Bretagne Atlantique, 35042 Rennes Cedex, France
}

\begin{abstract}
Multicarrier modulation systems suffer from large peak-to-average power ratio (PAPR). Tone reservation is a popular technique for PAPR reduction. It consists in reserving a number of carriers to produce a redundant additive signal which reduces the peak power. There are several schemes to select the reserved carriers. In this paper, we propose a new selection method for adaptive tone reservation. It is showed through simulations that this new proposed selection technique allows $5 \mathrm{~dB}$ gain in terms of signal-to-noise ratio for a bit error rate of $10^{-3}$, for different constellations, in frequency-selective Rayleigh fading channel.
\end{abstract}

Keywords-Tone Reservation (TR), Peak-to-Average Power Ratio (PAPR), Orthogonal Frequency Division Multiplexing (OFDM)

\section{INTRODUCTION}

Multicarrier modulation (MCM) systems are widely used in wireless and wireline communication applications; for example, discrete multi-tone modulation is used in the asymmetric digital subscriber line (ADSL) standard, and orthogonal frequency division multiplexing schemes (OFDM) are used in terrestrial digital television, $\mathrm{WiFi}$, and in the long term evolution (LTE). MCM systems have distinct advantages over single-carrier modulation systems, especially in a frequency selective fading channel, but they suffer from high fluctuations of the signal envelope. The efficiency of the high power amplifier in the transmission side, is then decreased because of these large variations. The peak-to-average power ratio (PAPR) is the random variable usually used to measure the variations of the MCM signals.

There are several PAPR reduction techniques such as partial transmit sequences approaches, selective mapping approaches [1], clipping and filtering techniques [2], and tone reservation (TR) techniques [3]. The latter family is one of the most popular schemes and it consists in using a set of reserved tones to design a peak cancelling signal for PAPR reduction. As TR works on reserved tones, no additional signal processing is required at the receiver side to extract the data information. Besides, the computation of the peak cancelling signal can be achieved thanks to an efficient convex optimization algorithm such as the second order cone programming [4], or a gradient project algorithm [5]. Due to these reasons, TR is quite popular for practical implementations and therefore seems to be a promising technique for commercial standards such as Digital Video Broadcasting-Terrestrial (DVB-T2). In the literature, there are several selection methods of the reserved tones in the TR reduction technique. The choice of the reserved tones can improves the TR performance as well as the performance of the system.

In this paper, we propose a new method to select the reserved tones in the TR method, in order to improve the bit error rate (BER) performance of the system, in the presence of a frequency selective fading channel. The selection of the reserved tones is adapted to the channel characteristics. Under the assumption that the channel state is perfectly known at the transmitter, the reserved tones are the ones that are the most affected by the channel. For the classical selection methods, the reserved tones are used to reduce the PAPR, their utility is then limited to this function. Our new selection technique make the reserved tones also useful for improving the BER performance of the scheme.

\section{ADAPtiVE TONE RESERVATION}

\section{A. Notations and definition}

Throughout this paper, an OFDM symbol $x(t)$ of duration $T_{u}$ is used and expressed as follows:

$$
x(t)=\sum_{m=0}^{M-1} X_{m} e^{j 2 \pi m F t}, 0 \leq t \leq T_{u},
$$

where $M$ is the total number of carriers, $F=\frac{1}{T_{u}}$ is the intercarrier space, $m F$ the $m$-th frequency and $X_{m}$ the input symbol carried out by the $m$-th carrier. After oversampling the signal by a factor $L, x_{l}$ such that $l=0, \ldots, L M-1$, are the discrete time domain samples at the instant $l T_{e}$ where $T_{e}=\frac{T_{u}}{L M}$ and $\mathbf{x}=\left[x_{0}, \ldots, x_{L M-1}\right]$ is the discrete OFDM symbol (vector). We will use capital letters to denote Fourier transform.

\section{B. Adaptive Tone Reservation (ATR) principle}

Instead of reserving the tones randomly, or according to a predefined set known at the transmitter and the receiver as in DVB-T2, they can be selected differently in order to improve the BER performance of the system. Our proposed method 
Table I: Coefficients of Channel C of Proakis.

\begin{tabular}{|l|c|c|c|c|}
\hline$h_{0}$ & $h_{1}$ & $h_{2}$ & $h_{3}$ & $h_{4}$ \\
\hline 0.027 & 0.460 & 0.688 & 0.460 & 0.027 \\
\hline
\end{tabular}

consists in selecting the carriers most affected by the frequency selective fading, and in using them to reduce the PAPR, rather than carrying useful information. Since these carriers will be discarded in the receiver side, the useful information will be carried in the less affected carriers, which helps improving the BER performance.

Thus, for every frequency selective fading channel $h$, the $M_{R}$ tones that correspond to the smallest $|H(f)|$ are reserved for the PAPR reduction process, according to Algorithm 1. We assume that there is a feedback of the channel state information from the receiver to the transmitter, and the channel is stationary during this period of time.

In order to understand this new selection solution, we consider in the first place a specific channel. Table I defines the frequency selective fading channel of Proakis $C$ [6]. The considered number of carriers, $M$, is equal to 1024 . Figure 1 shows the amplitude frequency response of the channel. Based on the observation of this figure, it can be seen that the channel has a spectral null around carrier indices 396 and 630 . The frequency coefficients around these indices are very small, and the relative noise around them is then very high.

By applying the new proposed selection method, the $M_{R}$ reserved carriers ( $M_{R}$ is equal to 12 is this case) are selected as described in Algorithm 1, and they are:

$$
\text { Reserved Tones }=[397,629,396,630,398,628,
$$$$
\text { 395, 631, 399, 627, 394, 632]. }
$$

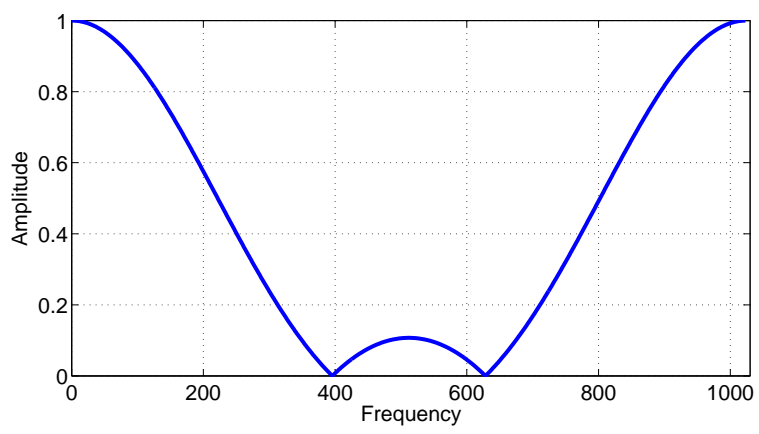

Figure 1: Amplitude Response of Proakis C Channel.

Let $\mathcal{R}$ be the set that contains the indices of the $M_{R}$ reserved tones. In the ATR method this set is obtained thanks to Algorithm 1, and in the TR approach this set is predefined as in the DVB-T2 standard. With this set of reserved tones, the main goal of the TR, and also the ATR approach is to find the effective peak cancelling signal in order to reduce the PAPR. Several analyses are proposed in the literature for this purpose [3], [4], [5]. In this paper, the TR method that uses the gradient project algorithm will be used to compute the signal to be added [5].

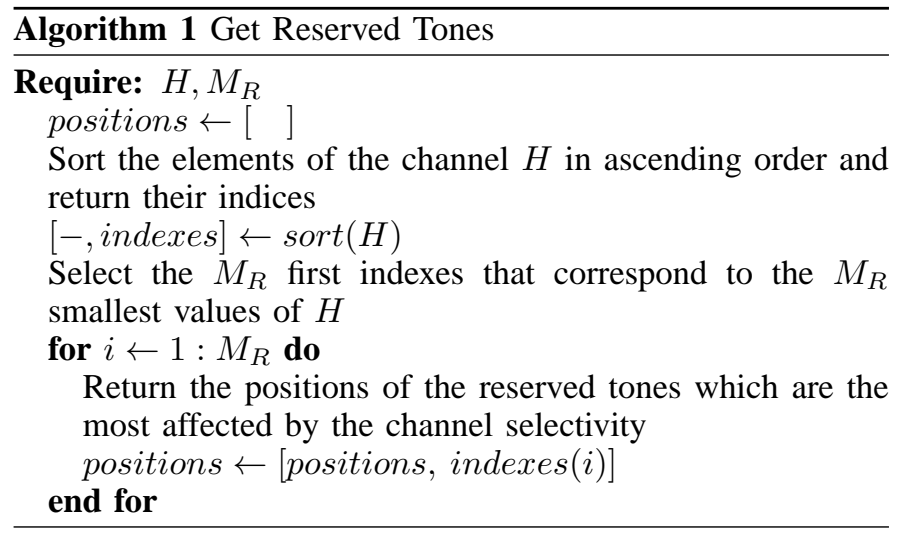

This method consists in maximizing the Signal to Clipping noise power Ratio (SCR). Let

$$
f(r)= \begin{cases}A e^{j \arg (r)}, & \text { if }|r| \geq A \\ r, & \text { else }\end{cases}
$$

denotes the clipping function, with $A$ a predefined threshold. Adding tones corresponds to replacing $x$ by $x+\mathbb{F}_{\mathcal{R}} \mathbf{C}$ where $\mathbb{F}_{\mathcal{R}}$ is the sub matrix of the inverse discrete Fourier transform matrix containing only the column indexed in the set of the reserved tones. The SCR to be maximized is defined as:

$$
\mathrm{SCR}=\frac{\|\mathbf{x}\|_{2}^{2}}{\left\|\mathbf{x}+\mathbb{F}_{\mathcal{R}} \mathbf{C}-f\left(\mathbf{x}+\mathbb{F}_{\mathcal{R}} \mathbf{C}\right)\right\|_{2}^{2}}
$$

Maximizing (4) is equivalent to minimizing its denominator by solving the following optimization problem

$$
\min _{\mathbf{C}}\left(\left\|\mathbf{x}+\mathbb{F}_{R} \mathbf{C}-f\left(\mathbf{x}+\mathbb{F}_{R} \mathbf{C}\right)\right\|_{2}^{2}\right) .
$$

Starting with the initial condition $\mathbf{c}^{(0)}=\mathbf{0}_{M_{R}}$, the peak cancelling signal can be computed iteratively thanks to the gradient project algorithm featuring the following equation [3]

$$
\mathbf{c}^{(i+1)}=\mathbf{c}^{(i)}-\mu \sum_{\left|x_{l}^{(i)}\right|>A}\left(x_{l}+c_{l}^{(i)}-A e^{j \arg \left(x_{l}+c_{l}^{(i)}\right)}\right) \mathbf{P}_{l}
$$

where $\mathbf{P}_{l}$ represents the $l$-th line of matrix $\mathbb{P}=\mathbb{F}_{R}\left(\mathbb{F}_{R}\right)^{H}$. Therefore, by adding the useful signal $\mathbf{x}$ in the two terms of (6), we obtain the reduced PAPR signal after the $(i+1)$ iterations as the following

$$
\mathbf{x}^{(i+1)}=\mathbf{x}^{(i)}-\mu \sum_{\left|x_{l}^{(i)}\right|>A}\left(x_{l}+c_{l}^{(i)}-A e^{j \arg \left(x_{l}+c_{l}^{(i)}\right)}\right) \mathbf{P}_{l},
$$

where $\mathbf{x}^{(i+1)}=\mathbf{x}+\mathbf{c}^{(i+1)}$. Note that, in the TR approach, because of the set of reserved tone positions is predefined (is the same for each $\mathbf{x}$ ), the matrix $\mathbb{P}=\mathbb{F}_{R}\left(\mathbb{F}_{R}\right)^{H}$ can be precomputed and stored. Therefore, the numerical complexity of the TR method is $\mathcal{O}(M L)$. In the ATR method, since the set of reserved is not predefined (it depends on the channel), the ATR method has the same complexity as the matrix $\mathbb{P}=\mathbb{F}_{R}\left(\mathbb{F}_{R}\right)^{H}$.

\section{RESULTS}

Simulations in this Section have been performed for OFDM with a number of carriers equal to $M=1024$, and a number of 
Table II: Channel Delay and Power Profile of ETU Channel.

\begin{tabular}{|l|c|c|c|c|c|c|c|c|c|}
\hline$\tau(n s)$ & 0 & 50 & 120 & 200 & 230 & 500 & 1600 & 2300 & 5000 \\
\hline$G_{\text {av }}(d B)$ & -1.0 & -1.0 & -1.0 & 0.0 & 0.0 & 0.0 & -3.0 & -5.0 & -7.0 \\
\hline
\end{tabular}

reserved tones equal to $M_{R}=12$ using 4QAM, 16QAM and 64QAM for BER performance, and 4QAM for PAPR analysis. The inter-carrier spacing is considered equal to $\Delta F=5 k H z$.

\section{A. BER Performance}

We evaluate the BER performance (in a frequency selective fading Rayleigh channel) of the proposed adaptive tone reservation (ATR) method based on adaptive tone selection, and compare it with a classical TR method which consists in reserving the first $M_{R}$ tones. The channel delay $\tau$ and the average path gain $G_{\mathrm{av}}$ that characterize the channel considered in these simulations are defined in Table II, which represents the extended typical urban (ETU) model used in the LTE multipath channel standard [7]. The gradient project algorithm is used in the TR simulations, with a number of iterations of $N=20$, stepsize $\mu=0.25$, and threshold $A$ corresponding to $6.5 d B$. The BER and the PAPR simulations are performed using $n_{1}=10^{4}$ and $n_{2}=10^{6}$ iteration loops respectively. Figure 2 gives the BER performance of the OFDM system based on the proposed ATR with adaptive tone selection, and compares it with the TR method with specific tone selection. As depicted in Figure 2, for a BER of $10^{-3}$, the proposed method gives a gain of $5 \mathrm{~dB}$ in terms of signal-to-noise ratio (SNR) for the different constellations used in the simulation (4QAM, 16QAM, 64QAM). This can be explained by the fact that the carriers affected by the deep fading of the channel do not carry useful information, and they are discarded in the receiver side. Therefore, they do not affect the BER of the whole system.

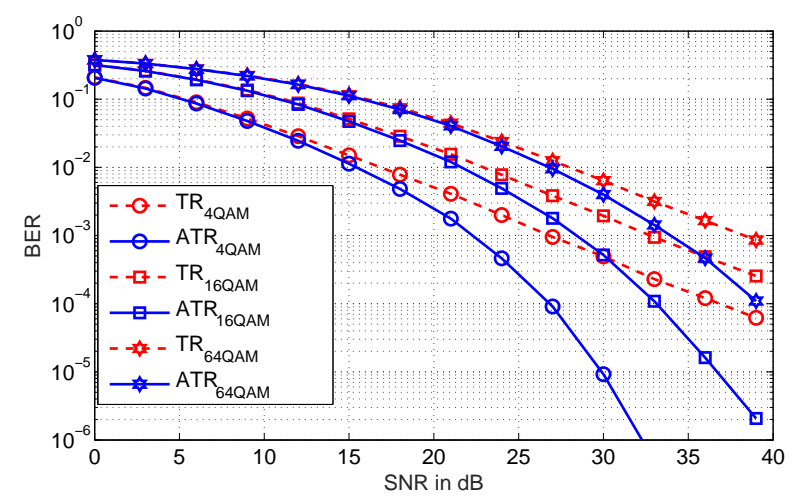

Figure 2: BER Performance of the Uncoded OFDM based on the Adaptive Tone Reservation for different constellations.

\section{B. PAPR Performance}

The proposed selection technique improves the BER in frequency selective fading channel, without reducing the PAPR performance compared with the classical method. In order to confirm this claim, we simulate the PAPR performance using the ATR reduction method based on the adaptive selection method and we compare it with a specific selection method which consists in reserving the first $M_{R}$ tones. The PAPR performance is evaluated using the complementary cumulative distribution function which is the probability that the PAPR exceeds a certain value $\gamma$. Figure 3 confirms that there is no degradation of the PAPR when the new selection method for ATR is used.

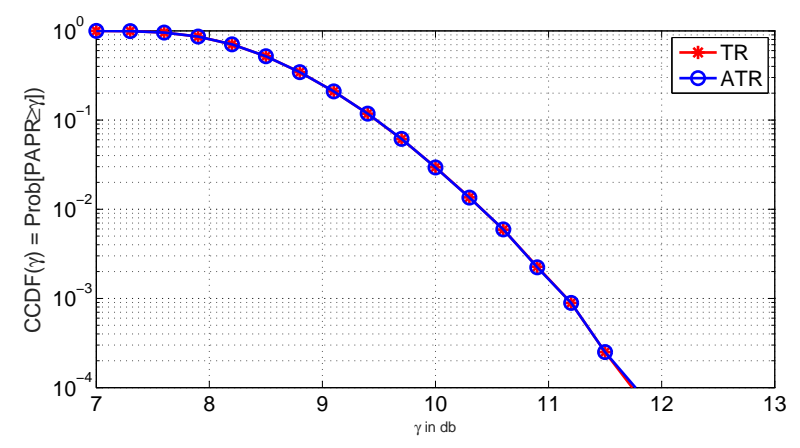

Figure 3: PAPR Performance Comparison between OFDM based on the Adaptive Tone Reservation and OFDM based on Classical Tone Reservation.

In this paper, we have proposed a new selection method for adaptive tone reservation. It has been shown through simulations that this new proposed selection technique allows $5 \mathrm{~dB}$ gain in terms of signal-to-noise ratio for a bit error rate of $10^{-3}$, for different constellations, in frequency-selective Rayleigh fading channel for uncoded OFDM system, without any degradation of the PAPR performance compared with a classical selection method.

The future work is about evaluating the gain in terms of BER performance for Coded-OFDM.

\section{ACKNOWLEDGMENT}

This work has received a French state support granted to the CominLabs excellence laboratory and managed by the National Research Agency in the "Investing for the Future" program under reference Nb. ANR-10-LABX-07-01.

\section{REFERENCES}

[1] R.W. Bauml, R.F.H. Fischer, and J.B. Huber. Reducing the Peak-toAverage Power Ratio of Multicarrier Modulation by Selected Mapping. Electronics Letters, 32(22):2056-2057, Oct 1996.

[2] Xiaodong Li and L.J. Cimini. Effects of Clipping and Filtering on the Performance of OFDM. In IEEE Communication letter, volume 2, pages 131-133, May 1998.

[3] J. Tellado-Mourelo. Peak to Average Power Reduction for Multicarrier Modulation. PhD thesis, Standford University, 1999.

[4] S. Zabre, Jacques Palicot, Y. Louet, and C. Lereau. SOCP Approach for OFDM Peak-to-Average Power Ratio Reduction in the Signal Adding Context. In Signal Processing and Information Technology, 2006 IEEE International Symposium on, pages 834-839, 2006.

[5] J. Tellado and J.M. Cioffi. Efficient Algorithms for Reducing PAR in Multicarrier Systems. In Information Theory, 1998. Proceedings. 1998 IEEE International Symposium on, pages 191-, Aug 1998.

[6] John G Proakis. Digital Communications. McGraw-Hill Inc, 2001.

[7] TS ETSI. 136101 V8. 23.0 (3GPP TS 36.101 V8. 23.0 Release 8). 